\title{
Amazones de tir dans la SF côté femmes!
}

\author{
Sylvie Bérard
}

\begin{abstract}
War-zones, Ama-zons: Sci-Fi on Women's Side!
Toward the end of the 1960s, when the cult of the holy trinity science-civilization-technology gives way to profound epistemological questioning, women find in the practices of science-fiction an unexpected terrain for formulating feminist concerns. In addition to allowing women who were previously relegated to the role of beautiful object to achieve the position of narrative and discursive subject, science-fiction "in the feminine" opens new avenues for the deployment of myth - particularly the myth of the Amazon. Sometimes transposed, sometimes revisited and transformed, the latter myth manifests itself in three different ways: as the crystalization of a sexual fantasy; as the incarnation of the warrior who vows an ancestral hatred of men; or as the only way of life possible in a society where men have disappeared. Feminist study of the figure of the Amazon is crucial insofar as it provides insight into the series of alternative models of society proposed by science fiction. Yet, as Bérard suggests, if the figure of the Amazon is enabling for feminism, that figure also assumes the polarization of subjects on the basis of their sex - an assumption that is not always compatible with the egalitarian aims of feminist projects.
\end{abstract}

The obvious absence of «sociological» men in feminist utopian fictions is not only a corrective rewriting of history but also testifies to the need to make a future different from the past.

- Frances Bartowsky, Feminist Utopias

Ponctuellement mais sûrement, le mythe de l'Amazone ressurgit dans les cultures occidentales. Fascinant est à observer l'intérêt que la littérature, toutes tendances confondues, porte à l'Amazone, depuis les tout premiers temps de l'Antiquité jusqu'à l'époque contemporaine. Utopie réjouissante pour les un-e-s, dystopie angoissante pour les autres, la société amazonienne a été représentée selon différents modes et à diverses fins. Il est jusqu'au féminisme radical et séparatiste à en avoir 
intégré certains modèles. La société amazonienne comme projet de renouveau social, comme modèle d'un état collectif idéal semble s'attirer la faveur dans la fiction féministe.

La science-fiction-SF pour les intimes - qui plonge volontiers dans la mythologie pour y puiser son étrange nouveauté (ou novum, comme dit Suvin), sait, elle aussi, exploiter le mythe de l'Amazone, soit pour le transposer, soit pour le revisiter et le transformer. Une part significative de la science-fiction féministe, issue en partie de la New Wave britannique et étatsunienne, revisite certains mythes dont celui de l'Amazone. Si, comme on l'a dit, «[1]es féministes de la SF trouvent dans le genre un terrain de choix pour exacerber leur militantisme, pour étudier et dénoncer les stéréotypes de la suprématie masculine» (Cordesse 165), les auteures de science-fiction ont décelé en la figure de l'Amazone un intérêt égal du point de vue littéraire engagé à celui qu'y ont trouvé, du point de vue social, leur consœurs féministes radicales partisanes de l'isolationnisme.

Ce qu'il est intéressant d'observer, dans la SF contemporaine produite par les femmes - et quand je dis $S F, j$ 'entends également utopie, les nuances génériques n'étant pas de circonstance ici - c'est non la reprise du mythe de l'Amazone mais sa réappropriation. Quand la science-fiction s'écrit par les femmes ou quand les féministes s'adonnent à la science-fiction, l'actualisation du mythe révèle parfois que, si dans une certaine mesure l'Amazone littéraire assure une revendication féministe, elle se fonde néanmoins sur une repolarisation parfois peut compatible avec les visées égalitaristes.

\section{La tentation mythique de la SF}

D'abord et avant tout, l'A mazone étant une femme mythique, l'observation de cette figure dans la SF sous-entend automatiquement deux questions: $1^{\circ}$ quelle place la SF fait-elle aux mythes; $2^{\circ}$ quelle place fait-elle aux femmes ou comment les femmes s'y taillent-elles une place.

«La science-fiction est la mythologie du monde moderne» (Le Guin 68), entend-on parfois. Aussi, l'utilisation du mythe de l'Amazone dans la SF côté femmes ne peut s'observer sans une certaine mise en contexte, les études existantes sur la SF indiquant que ce phénomène s'inscrit en quelque sorte dans le droit fil d'une tentation mythique généralisée dans la pratique comme dans l'étude de la SF.

Ainsi, Ursula K. le Guin considère elle aussi que le mythe en science- 
fiction a pour fonction de mieux nous faire comprendre le monde où nous vivons: "For science fiction does use the mythmaking faculty to apprehend the world we live in, a world profoundly shaped and changed by science and technology, and its originality is that it uses the mythmaking faculty on new material.» (Le Guin 70) Selon elle, il s'établit une nette distinction dans la SF entre la reprise du mythe et la création de nouveaux mythes, la première étant moins enrichissante que la seconde. L'analyse, cependant, dans l'un et l'autre cas, ne doit pas se résumer à un repérage des symboles.

Darko Suvin ne croit pas que la construction de mythes soit une avenue pertinente en SF. Il évoque les affinités «entre la science-fiction et le récit mythologique» (31-32), soulignant les rapports d'analogie qu'ils entretiennent sur le plan formel et morphologique. Toutefois, poursuitil, «il se peut que la fiction soit formellement ou morphologiquement analogue au mythe, mais en elle-même, elle n'est pas mythe. La fiction utilise des mythèmes à des fins non mythiques, et même antimythiques - sauf dans le conte merveilleux, le fantastique et la sous-littérature.» (32) La SF ramène le mythe à elle sans s'y conformer et sans tenter d'en générer, en résistant notamment au pouvoir de séduction du mythe en tant que modèle absolu: «En termes mathématiques, le mythe s'oriente vers des constantes, et la science-fiction vers des variables.» (33)

Le mythe, en SF comme dans toute production artistique, peut être traité de trois façons: $1^{\circ}$ "comme une sorte de vision symbolique corrélative au mode de la conscience mytho-poétique»; $2^{\circ}$ comme un récit, le récit étant traité comme un mythe; $3^{\circ}$ comme une étape de l'analyse, non comme une fin en soi (cf. Suvin 38-42). Darko Suvin considère que, selon ce dernier mode, on «doit encore résoudre les questions fondamentales de sa discipline, soit, pour la SF: est-ce que, et comment, ce mythe ou mythème a été transformé $1^{\circ}$ en fiction valable; $2^{\circ}$ en science-fiction valable.» (42) C'est cette dernière approche, envisageant le mythe comme une simple étape dans l'analyse de certains textes féministes de science-fiction, qui me semble la plus pertinente dans le cas de l'Amazone car cela permet de considérer le texte dans sa dynamique.

Pour ce qui est de la place des femmes dans la SF, sans doute que je n'en étonnerais pas beaucoup en annonçant que la SF a souvent réservé aux femmes la portion congrue. J'en estomaquerais sans doute plusieurs en soutenant exactement le contraire, c'est-à-dire en considérant que les femmes y trouvent de plus en plus leur compte. Et pourtant, une série 
d'exemples, durant les 25 dernières années, tendent à étayer cette seconde hypothèse. En ce sens, l'Amazone de SF est une figure séduisante que nombre d'auteures n'ont pas hésité à s'approprier. Disons que la figure de l'Amazone répond aux besoins les plus divers, qui ne sont pas toujours, mais qui peuvent s'avérer, féministes.

\section{L'Amazone au service de la SF}

S'appuyant sur Eschyle, la thèse de William Blake Tyrell sur l'Amazone comme exemple de la constitution des mythes grecs a deux visées.

La première consiste à démontrer que le mythe est fabriqué à partir de données culturelles sur des questions telles que la guerre, le sexe, l'ethnographie, la politique et, par-dessus tout, les rites touchant la transition, de l'enfance socialement définie, à l'âge adulte et au mariage. (xiii, je traduis)

La thèse de cet auteur suppose une construction patriarcale du mythe en même temps qu'elle implique une matriarchie inversée par rapport à l'ordre patriarcal: il s'agit «de replacer le mythe de l'Amazone dans le contexte de la création athénienne des mythes touchant le mariage.» (iv, je traduis) La lecture du mythe peut donc bel et bien s'avérer phallocentriste. Evelyne Reed, par exemple, considère que l'exacte réplique inversée du patriarcat contribue à expliquer le discrédit jeté sur le matriarcat en même temps qu'il ne rend pas compte avec justesse d'un ordre social différent du nôtre.

Une conception erronée - la 'loi' imposée aux hommes par les femmes, l'exacte réplique, mais inversée, de la domination masculine actuelle - contribue en partie à expliquer le discrédit jeté sur le matriarcat. Nombreux sont ceux, en effet, qui ne comprennent pas la nature, diamétralement différente, de l'ordre social primitif et du nôtre. (134)

Le mythe de l'Amazone pose un problème au niveau féministe. $D^{\prime}$ abord, en tant qu'espace du féminin, il est déjà mis en discours par une tradition littéraire et philosophique (cf. Jardine 36-37). L'exemple du sein coupé - le mythe dans le mythe - en est une illustration éloquente. Cependant, ce qui apparaît comme historiquement faux n'est pas nécessairement symboliquement non vrai. 
From the mythic point of view, it is right that the Amazon refused anything of the feminine or sexual that would handicap the defense of their autonomy. It is therefore probable that this fable of the "mutilated" breasts was historically false but symbolically true. (Paris 157)

Aussi, les fabulations autour de la fable nous renseignent-elles autant sur l'époque qui leur donne naissance que l'origine du mythe nous informe sur ses fondements. Et justement, le mythe de l'Amazone a souvent servi à exprimer la peur qu'ont les hommes des femmes beaucoup plus qu'à illustrer l'aspiration des femmes à l'indépendance.

En fait, l'analyse diachronique révèle que les représentations de l'Amazone tendent à des buts distincts selon les époques et les sociétés qui leur donnent naissance. S'attardant non à la fortune du mythe mais à son origine, il est possible de l'interpréter de manière féministe. Monique Wittig et Sande Zeig supposent que, historiquement, il dut y avoir un passage de l'image d'une femme préservant l'harmonie universelle à celle d'une femme n'assumant pas son destin de femme, si bien que les Amazones désormais ostracisées furent contraintes de préserver de force l'harmonie, d'où l'agressivité qu'on leur associe généralement (Wittig et Zeig 5). Les lectures et relectures du mythes exploitent tour à tour la vision d'une société pastorale et d'une collectivité farouchement guerrière.

[A]re Amazons enemies whom men must fight or equals with whom they may rightfully associate? In a patriarchal society, is it inevitable that any group of women who show a forcible desire to separate and establish well-defended boundaries be considered a threat? (Paris 158)

À cause sûrement de la nécessité de défendre un territoire, l'Amazone est souvent décrite dans sa fonction de combattante. Il est vrai que les Amazones historiques ou mythologiques se situent dans une position d'affrontement par rapport à l'Autre majoritairement masculin. C'est aussi ce qui motive l'appellation d'Amazone qu'on accole parfois par métaphore à certaines femmes s'étant distinguées historiquement. Dans son encyclopédie des Amazones, Jessica Amanda Salmonson fait du combat l'aspect essentiel de cet archétype. Pour les besoins de son ouvrage, l'Amazone est prise dans son sens de duelliste ou de soldate, 
par dessein ou par la force des choses, chevaleresque ou cruelle, qui s'engage dans des combats directs avec l'Autre (cf. Salmonson xi). L'auteure s'inspire ainsi de l'A mazone de la mythologie grecque, adoratrice d'Artemis, qui est guerrière par nature, habile dans le maniement d'armes ou dans les affrontements corps à corps.

Concuremment à l'interprétation du mythe comme leçon patriarcale ou comme résidu matriarcal, on a donc aussi une lecture du mythe qui va dans le sens d'un comportement guerrier ou, du moins, d'une attitude défensive. Mais si on y regarde de plus près, le combat apparaît plus comme un symptôme que comme une caractéristique en soi. Il semble plus juste de définir l'Amazone dans le type de rapport qu'elle entretient avec l'Autre - l'Autre étant dans ce cas le «mâle de son espèce», pour paraphraser Louky Bersianik - que ce rapport se traduise par le combat direct ou par une résistance plus implicite, physique ou psychologique.

\section{Modes de réappropriation de l'Amazone}

Dans les œuvres quej'ai étudiées, la figure de l'Amazone est donc posée surtout dans son rapport à l'Autre antagonisé. Le mythe est actualisé selon trois modes de récupération, de la soumission finale à l'éviction pure et simple, en passant par la résistance acharnée.

Selon un premier mode, l'Amazone (que je baptise Amazone érogène, on va voir pourquoi), comme objet du désir ou sujet désirant, doit lutter contre un double bind inextricable: elle n'est désirée par l'Autre que lorsqu'elle résiste à l'Autre; si elle désire l'Autre, se soumettant à l'Autre elle cesse d'être elle-même et n'est plus désirée par l'Autre. C'est que l'Amazone est attirante justement dans sa résistance à l'autre.

On the part of the "Amazonian" woman, the double bind occurs when she falls in love with a man. The more she resists, the more he admires her, for it is the Amazon in her that he loves. But if she yields to him, she is no longer an Amazon, becoming instead an "ordinary" woman: she loses his love the moment that, through love, she becomes vulnerable. She has no choice but to fight or be overcome. (Paris 158)

Dans ce cas, lorsque l'Amazone cède - à ses propres pulsions ou à l'Autre-différents indices laissent parfois croire qu'elle revient à sa vraie nature, à sa «féminité reniée» (Moore 105) ou qu'elle franchit le cap de 
l'âge adulte. Une bonne partie de l'œuvre de Catherine L. Moore illustre assez bien cette situation, comme le montre cet exemple tiré de La nuit du jugement paru en 1952:

Soudain, elle prit peur. Cette émotion qui les liait était une drogue plus forte et plus dangereuse que le vin... c'était la chose la plus terrible qui pouvait arriver à une Amazone. Lui aussi pouvait essayer de l'influencer - et elle fut prise d'une crainte désespérée d'être incapable de lui résister. (140)

Dans la nouvelle «Une fille un peu démodée» de Joanna Russ, une Amazone dont le métier est de tuer des hommes résout la question en s'offrant en guise d'appareil domestique un simulacre qui ne possède de l'homme que les fonctions sexuelles:

L'ordinateur central transmet alors une série de signaux aux implants dans son cerveau et il s'étend avec obéissance sur le lit. Lorsque je dis à l'ordinateur central: «Dors.», Davy s'endort. C'est une merveilleuse excroissance de la maison. Le protoplasme originel provenait d'un chimpanzé, je crois, mais le comportement n’est plus contrôlé organiquement. (250-251)

Cela m'amène à ma deuxième catégorie que j'ai nommée Amazone castrante. Celle-ci, dans sa fonction revendicatrice voire guerrière, maintient l'Autre à distance, refusant tout élément social ou sexuel contraignant. Elle fuit purement et simplement la compagnie des hommes. On la retrouve surtout dans certaines utopies ou uchronies féministes où les hommes sont, sinon carrément écartés (Monique Wittig, Les Guérillères), du moins sont évincés, ou conservés aux fins de reproduction (Joanna Russ, L'Autre Moitié del'homme). Les femmes, dans ces romans, ne pactisent pas avec les hommes, et les hommes qui se trouvent en travers de leur chemin n'ont qu'à bien se tenir.

Le Patron était en colère à cause de son érection, assez en colère pour deux, etj'entrai moi-même en érection-jeveux dire par là que les muscles greffés sur mes doigts et mes mains rétractèrent la peau avec cette démangeaison caractéristique [...]. Je lui ratissai joyeusement le cou et le menton, et, lorsqu'il m'étreignit avec colère, je lui plantai mes griffes dans le dos. (AM 247)

Un personnage d'Élisabeth Vonarburg, judicieusement prénommé Judith - à titre indicatif, dans la mythologie judéochrétienne, l'héroïne 
du Livre de Judith, pour sauver la ville de Béthulie, séduit Holopherne et, l'ayant fait boire, lui tranche la tête-tient à peu près le même discours:

«De bonne foi? J'espère tout de même qu'il n'est pas assez stupide pour croire ça!» Judith fait une moue, le visage durci: «C'est eux ou nous. Il n'y a pas de cohabitation pacifique possible. Ils ne nous laisseront jamais de bon gré les commandes qui nous reviennent, puisque nous sommes les plus nombreuses....» (SC 233)

Dans certains exemples moins... radicaux, comme dans «Le vaisseau survie» de chez Judith Merrill, la proportion des hommes est maintenue artificiellement très faible par rapport à la population féminine, de manière à assurer - eh oui - l'harmonie.

Dans la troisième catégorie, que j'ai appelée Amazone déserte, tout combat est écarté. L'Amazone classée sous cette dernière rubrique n'est qu'une catégorie commode puisqu'il s'agit en fait de la femme vivant en communauté de femmes ou gynécée parce que c'est le seul endroit tolérable dans le cas de sociétés totalitaires comme dans La Servante écarlate de Margaret Atwood, possible dans le cas de sociétés où les hommes ont disparu pour des raisons biologiques comme dans Chroniques du pays des mères d'Élisabeth Vonarburg ou imaginable dans le cas de communautés planétaires féminines où l'absence du mâle humain n'est ressentie qu'à partir du système de référence terrien.

Cette catégorie permet d'observer que l'Amazone (si Amazone il y a), lorsqu'elle n'est pas représentée dans son rapport à l'Autre, instaure de nouveaux paradigmes non déterminés par le masculin et le féminin. La société que Joanna Russ construit sous le nom de Lointemps - Whileaway dans la version originale - l'illustre bien. Les hommes, sur cette planète, ont tous disparu; six siècles plus tard, les femmes évoluent dans une société qui n'a plus aucune commune mesure avec celle qu'on connait. Quand des hommes débarquent tout à coup (dans la nouvelle "Quand ça change», mais non dans l'Autre moitié de l'homme), c'est presque en extra-terrestre: ils s'étonnent que les femmes soient mariées et demandent à voir des «gens». L'incompréhension suscite des quiproquos savoureux:

Finalement, il ajouta: «Dans l'univers d'où je viens, les femmes ne s'habillent pas aussi simplement.» «Comme vous? demandai-je. Comme une mariée?» Car ces hommes portaient de l'argent de la tête aux pieds. (QC 359) 
De même, dans la nouvelle de Christine Renard «Les narcisses poussent le soir", l'équipe d'exploration terrienne se voit contrainte de réviser successivement sa lecture de la société vivant sur Andromède III. D'abord, les Vris, ces créatures qu'on croyait à prime abord socialement indifférenciées se révèlent en fait être sexuellement indifférenciées: «Tous... je devrais dire «toutes», car leur morphologie était indubitablement féminine [...].» (134) Et alors que la parthénogénèse est le modèle de sexualité qui apparaît le plus probable d'un point de vue terrien, les recherches démontrent bientôt que la reproduction des Vris se réalise par une sexualité narcissique, sorte d'hermaphrodisme virtuel: «Des miroirs, elle n'en verrait pas avant d'avoir l'âge de perdre sa virginité avec son reflet bien-aimé.» (139)

\section{Splendeurs et misères de l'Amazone science-fictionnelle}

Ces nouvelles où la rencontre avec une colonie amazonienne s'actualise après des années $\mathrm{d}$ 'isolement - ou un isolement absolu - insistent toutes sur une question: notre appréhension de l'autre se fait d'abord à partir de notre propre système de référence. Cependant, que l'Amazone conserve des rapports, même tendus à l'extrême, avec l'Autre ou qu'elle s'en trouve éloignée sciemment ou par la force des choses, elle est tout aussi fascinante dans l'optique d'une lecture féministe de la SF. Si en science-fiction, lors du contact avec $l^{\prime}$ Autre, on a le choix entre: $1^{\circ}$ reconnaissance et cohabitation; $2^{\circ}$ soumission et fusion; $3^{\circ}$ dénégation et affrontement (cf. Cordesse 134), $\mathrm{c}^{\prime}$ est résolument cette troisième solution qui est adoptée par la société de l'Amazone, quelle qu'elle soit.

La figure de l'Amazone contient donc en germe une certaine représentation de l'altérité. Cette dernière en SF peut bien sûr avoir différents visages: «there is the sexual Alien, and the social Alien, and the cultural Alien, and finally the racial Alien» (Le Guin 93), mais il demeure que l'Autre en science-fiction est souvent postulé comme non-humain. Or, en termes science-fictionnels, c'est la femme qui souvent est posée comme l'extra-terrestre dans son propre monde, comme l'alien dans un monde aux paradigmes masculins. Il n'est d'ailleurs pas rare que, dans la production récente de science-fiction féministe, la figure de l'alien soit utilisée par analogie pour décrire la position des femmes dans les sociétés occidentales: «In recent feminist science fiction the alien is used to comment on the position of women in contemporary Western society; after all, women are aliens in a world in which humanity is described as 
masculinity.» (Cranny-Francis 194) Prenant le parti de l'Amazone, et adoptant un point de vue qui est le sien, les auteures opèrent ainsi un renversement par lequel l'homme devenu Autre est observé à travers la lorgnette d'un ordre symbolique amazonien. Dans ces fictions, les femmes ont la parole et la transmettent de mère en fille: «Here the women are portrayed as speakers, knowers, and bearers of fables. They change from readers of orthodoxy to writers of texts against the canon.» (Bartkowski 38-39)

On rencontre d'ailleurs certaines fictions, hybrides par rapport aux catégories précédemment établies, qui reposent sur la cohabitation/ confrontation de différents types de sociétés amazoniennes voire de différents types de sociétés tout court. Cette rencontre dialectique de diverses identités sexuales (j'emprunte ce néologisme à Guy Bouchard 35-4) révèle parfois, au détour d'une fiction, à quel point les identités sont le fruit d'une construction sociale.

Dans L'Autre Moitié de l'homme, roman appartenant au même cycle que «Quand ça change» et «Une fille un peu démodée», la planète Lointemps n'est que l'une des quatre sociétés parallèles en présence:

[N]ous rencontrons tour à tour Jeannine l'aliénée femme-objet, Janet l'habitante du pays sans hommes, Jael la tueuse d'hommes dans un monde divisé en deux pays, celui des hommes et celui des femmes, enfin, Joanna la contemporaine qui réunit malaisément ces aspects d'elle-même. (Cordesse 167)

Dans ce récit, Joanna Russ confronte, non sans humour, différents points de vue sans établir véritablement de hiérarchie. Au niveau de la narration, les quatre voix en viennent à fusionner: "J'ai dit adieu et moi, Janet, je suis partie avec Laur; et moi, Joanna, je les ai aussi regardées s'éloigner; et je suis allée faire visiter la ville à Jael, moi, Jeannine, moi, Jael, moi-même.» (AM 288) Pire: alors que Janet l'habitante du pays sans homme vit dans la certitude que les hommes ont disparu suite à un fléau, Jael la tueuse d'hommes lui laisse entendre que le fléau aurait pu être secrètement provoqué!

Élisabeth Vonarburg réserve un traitement similaire à l'Amazone, qui n'est dans Le Silence de la cité qu'un des modes de vie possible sur une terre ravagée. Comme le souligne David Ketterer, l'auteure fait cohabiter les deux visions potentiellement antagonistes de l'intégration sexuelle et du séparatisme. Le personnage central n'est pas Judith l'Amazone mais Élisa, une mutante qui possède la faculté de changer 
de sexe à volonté et, par conséquent, une capacité d'émulation psychologique qui lui fait redouter la disjonction entre les classes de sexe. Cependant, comme elle considère l'envers des choses, elle n'est même pas certaine non plus que Judith ait tort! On a relevé dans cette œuvre «l'aspiration de l'humanité à dépasser la différenciation pour atteindre à la fusion. De cette fusion naît la promesse d'une humanité toujours nouvelle» (Gadbois 45-50), comme l'illustre l'extrait suivant:

[...] Ah, Dieu et le Diable. Ce serait tellement plus facile s'ils étaient aussi nettement distincts. Le blanc, le noir, le bien, le mal... et tiens, Hanse et Élisa. Manilo et Judith pouvaient croire à leur antagonisme, ils habitaient des corps séparés. Mais Élisa n'a pas cette échappatoire. Elle est Hanse, et Élisa, ni Dieu ni Diable, avec toutes les capacités de son corps à métamorphoses, elle est... un être humain, ni aussi faillible ni aussi infaillible qu' elle voudrait bien le croire. (SC 275)

Le doute, le questionnement, l'ébranlement des certitudes, voilà bien ce que la science-fiction la mieux tournée vise à susciter chez les lecteurs et les lectrices. La science-fiction est distanciation, non téléologie. Que la figure de l'Amazone, dans une réappropriation plurielle, ébranle les certitudes patriarcales, et la science-fiction féministe aura atteint une partie de ses objectifs.

Dans le sillage de la New Wave, alors que la science fait place à une spéculation tous azimuts, les femmes trouvent en science-fiction un terrain inattendu pour déployer leurs propres préoccupations. L'Amazone n'est pas la seule figure de femme résolument sujet dans la science-fiction - qui a aussi livré des femmes messies, prêtresses, leaders politiques - mais elle est une figure importante. Dans sa version féministe, elle permet d'actualiser des représentations différentes de celles véhiculées dans la science-fiction traditionnelle, différentes en tout cas de l'image de la femme que livre Jacques Sadoul, cité par Marianne Leconte: «Joli lot de Terriennes. Elles peuvent servir d'épouse, de maîtresse, d'esclave» et «De nourriture» (262).

La science-fiction dite au féminin ouvre des avenues nouvelles pour le déploiement du mythe en général et de celui de l'Amazone en particulier. Toutefois, l'observation de l'Amazone dans la SF au féminin, si elle renseigne sur une figure essentielle d'un éternel féminin bien particulier, ne permet pas toujours de rendre compte de l'ensemble de l'œuvre où 
elle est employée. Tout bien considéré et même si la fiction n'est ni un mode d'emploi ni un traité de mythologie, la SF livre plus d'informations sur l'Amazone que cette dernière n'en livre sur la SF. Autrement dit, le repérage de la figure de l'Amazone n'est qu'une étape dans la lecture de ces œuvres, dont le discours gagnerait subsquéquemment à être analysé.

Il est cependant intéressant d'observer que la communauté de l'Amazone n'est pas toujours représentée comme une solution idéale à la société patriarcale lorsqu'elle demeure le reflet symétrique de cette dernière, se résumant ainsi à la fois au fantasme et au contre-fantasme par excellence produit par l'ordre symbolique dominant. Il est vrai que l'Amazone littéraire, selon toute vraisemblance, renforce surtout la polarisation des identités. Or, la science-fiction contemporaine, plutôt que de contester les paradigmes couramment admis, vise surtout à s'en extirper.

These narratives, quite simply, are telling us that we had better start speaking the same language, lest Mother Eve, the Blessed Virgin, and the legion of uncanonized housewife saints permit our species to do what none has ever done before: sever one half from the other, lose the common language, and tacitly declare itself extinct. (Arbur 90)

C'est sans doute pourquoi, peut-être, la version féministe de cette SF préfère souvent à l'Amazone la solution ou la pirouette de la fusion sexuelle par androgynie ou hermaphrodisme - voire de la reproduction asexuée.

Côté jardin, la société amazonienne, en tant que modèle d'un projet social, apparaît très fructueuse du point de vue féministe, et différentes auteures de SF en ont exploité les avenues durant plusieurs années. Côté cour, dans certaines fictions contemporaines, la figure de l'Amazone sert de repoussoir, de preuve par l'absurde que l'avenir du futur est ailleurs que dans l'antagonisation des identités, hors des rôles stéréotypés, même renversés par rapport au construit social.

* Cet article est la version remaniée d'une communication présentée au Colloque de l'Association des professeurs de français des universités et des collèges canadiens (APFUCC), Congrès des Sociétés savantes, Ottawa, juin 1993. 


\section{Ouvrages cités}

Arbur, Rosemarie. «Fights of Fancy: When the 'Better Half' Wins» dans Slusser, George et Eric S. Rabkin (dir.), Fights of Fancy. Armed Conflict in Science Fiction and Fantasy, Athens/London: University of Georgis Press, 1993.

Atwood, Margaret. La Servante écarlate, Paris: Robert Laffont, 1987.

Bartkowski, Frances. Feminist Utopias, Lincoln/London: University of Nebraska Press, 1989.

Bersianik, Louky. L'Euguélionne. Roman triptyque, Montréal: La Presse, 1981.

Blake Tyrell, William. Amazons. A Study in Athenian Mythmaking, Baltimore/London: The John Hopkins University Press, 1984.

Bouchard, Guy. «Androgynie et utopie», dans Pelletier, Lise et Guy Bouchard, Féminisme et androgynie: Explorations pluridisciplinaires, Sainte-Foy: Les Cahiers du GRAD, 1990.

Cordesse, Gérard. La Nouvelle Science-fiction américaine, Paris: Éditions Aubier Montaigne, 1984.

Cranny-Francis, Anne. Feminist Fictions, New York: Saint Martin's Press, 1990.

Gadbois, Vital. «Phénix, Protée ou Janus», Imagine..., 22.

Jardine, Alice A. Gynésis. Configurations de la femme et de la modernité, Paris: Presses universitaires de France, coll. «Perspectives Critiques», 1991.

Letterer, David. Canadian Science Fiction and Fantasy, Bloomington/Indianapolis: Indiana University Press, 1992.

Le Guin, Ursula K. "On fantasy and science fiction», The Language of the Night, London: Women's Press, 1989.

Leconte, Marianne. «Une Autre, Femme», Femmes au futur, Marabout, 1976.

Merrill, Judith. «Le vaisseau survie», Histoires de sexe-fiction, Paris: Le livre de poche, coll. «La grande anthologie de la science-fiction», 1985.

Moore, Catherine, L. La nuit du jugement, Paris: J'ai lu, 1966 [1952].

Paris, Ginette. Pagan Meditations. The World of Aphrodite, Artemis, and Hestia, Dallas: Spring Publications, 1986.

Reed, Evelyn. Féminisme et anthropologie, Paris: Denoël/Gonthier.

Renard, Christine. «Les narcisses poussent le soir», dans Renard, Christine et Claude Chenisse, À la croisée des parallèles, Paris: Denoël, 1981. 
Russ, Joanna. L'Autre moitié de l'homme, Paris: Presses-Pocket, coll. «SF», 1977. Dans le texte, cette référence est identifiée $A M$.

—. "Quand ça change», Histoires de sexe-fiction, Paris: Le livre de poche, coll. «La grande anthologie de la science-fiction», 1985. Dans le texte, cette référence est identifiée $Q C$.

—. «Une fille un peu démodée», Histoires de sexe-fiction, Paris: Le livre de poche, coll. «La grande anthologie de la science-fiction», 1985. Dans le texte, cette référence est identifiée $F D$.

Salmonson, Jessica Amanda. The Encyclopedia of Amazons, New York: Anchor Books Doubleday, 1991.

Suvin, Darko. Pour une poétique de la science-fiction, Montréal: Presses de l'Université du Québec, 1977.

Vonarburg, Élisabeth. Chroniques du pays des mères, Montréal: Québec/Amérique, 1992.

_. Le Silence de la cité, Paris: Denoël, coll. «Présence du futur», 1981. Dans le texte, cette référence est identifiée $S C$.

Wittig, Monique. Les Guérillères, Paris: Minuit, 1969.

Wittig, Monique et Sande Zeig. Brouillon pour un dictionnaire des amantes, Paris: Grasset/Fasquelles, 1976. 